\title{
Assessment of Cost-Effectiveness on Remote Monitoring for Cardiovascular Devices in Japan
}

\author{
Tomoya Shirane ${ }^{1}$ \\ ${ }^{1}$ School of Graduate Studies, Open University of Japan, Chiba, Japan \\ Correspondence: Tomoya Shirane, School of Graduate Studies, Open University of Japan, Chiba, Japan. Tel: \\ 81-(0)43-276-5111. E-mail: tomoya.shirane@gmail.com
}

Received: August 2, 2019 Accepted: August 25, 2019 Online Published: September 1, 2019

doi:10.5539/gjhs.v11n11p1

URL: https://doi.org/10.5539/gjhs.v11n11p1

\begin{abstract}
Background: 2018 revision of the health insurance reimbursement in Japan brought additional fee for Cardiovascular Implantable Electronic Devices (CIEDs) Management by Remote Monitoring. The adaption of CIEDs Remote Monitoring has already been recommended by the societies, but the cost-effectiveness evaluation about the system has not been enough. This research was designed, therefore, to evaluate the cost-effectiveness about CIEDs Remote Monitoring in Japan.

Methods: A systematic review was conducted along with Preferred Reporting Items for Systematic Reviews and Meta-Analyses (PRISMA). Certain criteria and search strategy were pre-defined to identify studies that could be included into this research. The process of the quality assessments was planned by Critical Appraisal Skills Programme (CASP). Quality Adjusted Life Years (QALYs) that were extracted from the included studies would be calculated into Incremental Cost-Effectiveness Ratio (ICER) with the reimbursement amount in Japan.

Results: Three studies met the systematic review criteria after the selection along with PRISMA flow diagram. The quality of the included studies was assured by CASP Checklists designed for RCT and Cohort Study. ICERs from the selected studies were provided as 569,697 JPY, 1,220,000 JPY, and 311, 111 JPY for the patient groups enrolled with Remote Monitoring system.

Conclusion: ICERs for CIEDs Remote Monitoring were demonstrated as the cost-effective under the threshold set by Central Social Insurance Medical Council (Chuikyo). As this study put the validity of the cost-effectiveness approach in a certain field in Japan, this kind of evaluation should be performed on more areas along with the guideline by Chuikyo.
\end{abstract}

Keywords: cost-effectiveness, CRT-D, ICD, pacemaker, remote monitoring, telemedicine

\section{Introduction}

There have been over 15 years since the remote monitoring system for Cardiac Implantable Electronic Devices (CIEDs) was first introduced. The technology has been developed dramatically for years, and now, although the features might vary based on the manufactures, the system becomes highly sophisticated, such as transmitting the data automatically as daily or weekly basis, detecting clinical events and sending the related alerts, making the patients transmit their health data manually and so on.

Remote Monitoring has been recognized as the recommended system for the patients who are implanted with CIEDs by the major societies in the world. Heart Rhythm Society (HRS) in the United States published Expert Consensus Statement in 2015 during the Heart Rhythm Society's 36th Annual Scientific Sessions. The statement recommended Remote Monitoring as a standard of care for patients with CIEDs by putting Class of Recommendation with Level of Evidence (Slotwiner et al., 2015). European Society of Cardiology (ESC) updated Heart Failure Guidelines in 2016, which included "multiparameter monitoring based on ICD (IN-TIME approach) may be considered in symptomatic patients with HFrEF", even as Class IIb, under Recommendations for exercise, multidisciplinary management and monitoring of patients with heart failure (Ponikowski et al., 2016). Japanese Heart Rhythm Society (JHRS) announced the recommendation on the webpage, along with Expert Consensus Statement by HRS in 2015, that the benefits of Remote Monitoring could be considered as high and the installation to patients with CIEDs would be recommended as a standard management approach (Japanese Heart Rhythm Society, 2016). And Japanese Circulation Society (JCS) and JHRS jointly updated the Guideline on 
Non-Pharmacotherapy of Cardiac Arrhythmia in 2019, which included CIEDs Remote Monitoring based on the workflow developed within the hospitals as Class I with Evidence Level B (Kurita \& Nogami, 2019).

Although Remote Monitoring for CIEDs is, with the recommendations by the major societies from the world, now well accepted as the standard of care for the patients implanted with these devices, the health economics evaluation on this system has been under-developed, and the evidence around this topic has not been established yet (Shoda, 2012).

Also, the reimbursement amount for Remote Monitoring with CIEDs in Japan had been considerably low by thinking of the workload and resources that would be required. The monthly fee for Remote Monitoring was 600 Japanese Yen (JPY) per patient per month before 2018. The large, centralized institutions, such as National Cardiovascular Center, would put the workflow with some dedicated personnel to manage those patients effectively and efficiently (Kusano, 2017), but many hospitals are unable to take that approach due to the low reimbursement.

Under these circumstances, as the lobby activities by the related societies into the Ministry of Health, Labor, and Welfare (MHLW) might make some impacts, the reimbursement for CIEDs Remote Monitoring was increased to 3,200 JPY per patient per month by 2018 Reimbursement updates, almost 5 times higher than before. The additional amount for Remote Monitoring at this time could be controversial, as there seems to be no justification based on the cost effectiveness analysis.

Therefore, this study was designed and conducted to evaluate the cost-effectiveness of CIEDs Remote Monitoring based on the reimbursement volume updated in 2018 by utilizing Quality Adjusted Life Years (QALYs) measurements and Incremental Cost-Effectiveness Ratio (ICER) calculation.

\section{Methods}

\subsection{Eligibility Criteria}

A systematic review was performed along with Preferred Reporting Items for Systematic Reviews and Meta-Analyses (PRISMA) in order to identify the studies that can be included into this cost-effectiveness analysis. Central Social Insurance Medical Council (Chuikyo), an advisory board about Japanese health insurance and reimbursement system under MHLW, published a guideline in 2015, so-called Analysis Guidelines of Cost-Effectiveness Evaluation under Central Social Insurance Medical Council, which requires the cost-effectiveness analysis to be done by the systematic review methodology if the additional efficacy and/or safety is to be evaluated (Fukuda, 2015).

In order to identify trials relevant to this research topic, a certain search strategy was performed by using pre-determined inclusion and exclusion criteria described in Table 1. Included participants were those with CIEDs implanted and followed up equal to or longer than 12 months. Included studies were all prospectively designed with CIEDs patient management involved, and contained QALYs measurable in order to analyze ICER perspective. All the publications before 1971 were excluded as the telemonitoring technology of pacemaker through telephone line (trans-telephonic monitoring, TTM, which might be considered as a prototype of the current Remote Monitoring system) was first introduced in that year (Slotwiner et al., 2015).

Table 1. Inclusion and exclusion criteria for identifying relevant studies via search strategy

\begin{tabular}{ll}
\hline Inclusion Criteria & Exclusion Criteria \\
\hline $\begin{array}{l}\text { 1. Studies involving CIEDs such as Implantable } \\
\text { Cardioverter Defibrillator, Cardiac Resynchronization } \\
\text { Therapy, or Pacemaker. }\end{array}$ & 1. Studies not involving CIEDs \\
\hline 2. Follow Up period longer than or equal to 12 months & 2. Follow Up period shorter than 12 months \\
\hline $\begin{array}{l}\text { 3. Prospective studies involving CIEDs patient management } \\
\text { by Remote Monitoring system }\end{array}$ & 3. Not prospective studies \\
\hline $\begin{array}{l}\text { 4. QALYs available for ICER evaluation } \\
\text { 5. Published in or after 1971 }\end{array}$ & 4. QALYs unavailable or no health economics evaluation \\
\hline
\end{tabular}




\subsection{Search Strategy}

The literature search was conducted in September 2018, on the databases of MEDLINE (via PubMed), Springer, CiNii, J-Stage, and JAIRO. The search was limited to English and Japanese languages only. The following search keywords and terms were applied to find relevant papers: remote monitoring, health economics, quality of life, and pacemaker in English, and remote monitoring and health economics in Japanese. The Boolean operators used were $A N D$ and $O R$. The searches on keywords or terms were performed on all the selected databases and on the complete articles, including the title, summary, text, keywords, and so on. Specifically, for example, the search on PubMed was performed on [All Fields].

The first screening was carried out by eliminating all the duplications from the exported list of the searched publications. The next step was to review the overview of the publications by looking into their abstracts or summaries in order to exclude the articles that would not be suitable for this study. The final step was undertaken by reviewing the full texts of the screened publications in order to determine the eligibilities in detail.

\subsection{Quality Assessment}

The quality and risk of bias assessment of included articles were carried out using the Critical Appraisal Skills Programme (CASP). The checklists used for the articles included into this study were CASP Randomised Controlled Trial Checklist (Critical Appraisal Skills Programme (CASP), 2018b) and CASP Cohort Study Checklist (Critical Appraisal Skills Programme (CASP), 2018a).

\subsection{Data Extraction}

Reported measures of QOL were extracted as QALYs scores. Also, the study backgrounds and patient population were evaluated, mainly for the purpose of the quality and risk assessment, such as study characteristics (authors, year of publication, country, study duration, sample size, age, sex, NYHA class, LVEF, implantation devices such as pacemaker, defibrillators, and/or cardiac resynchronization therapy) and the major outcomes.

\subsection{Incremental Cost Effectiveness Ratio (ICER) Analysis}

QALYs from each study were evaluated by being combined with the reimbursement system in Japan from 2018 to 2020. Specifically, the reimbursed amount as addition by CIEDs follow-up through Remote Monitoring system is now 320 points as of the update in 2018. The reimbursement amount of CIEDs traditional follow-up as face to face has been set as 360 points historically. The reimbursement point is calculated by 10 JPY per 1 point. These calculations were applied to each study result. Therefore, one follow-up by the traditional method as face to face was considered as 3,600 JPY. And it was assumed that the remote system was monitoring the patients throughout the follow-up period of the studies, so the remote method was calculated as 3,200 JPY each month. And the calculated ICER from each study was eventually compared with the threshold set by Chuikyo.

\subsection{Handling Uncertainty of Cost-Effectiveness Analysis}

One-way sensitivity analysis was conducted to evaluate how Remote Monitoring reimbursement fee would affect ICERs that were calculated by QALYs extracted from the included studies.

\section{Results}

The literature search identified 1,693 from the selected databases through the internet, which were reduced to 1,645 once the duplicates were removed. By applying the inclusion and exclusion criteria, the number of studies were further reduced to 37 . After the full texts of 37 potentially relevant studies had been reviewed, 3 articles met the selection criteria finally, as described in Figure 1, and were included into this study (Zanaboni et al., 2013; Ricci et al., 2017; Lopez-Villegas, Catalan-Matamoros, Robles-Musso, Bautista-Mesa, \& Peiro, 2018). 


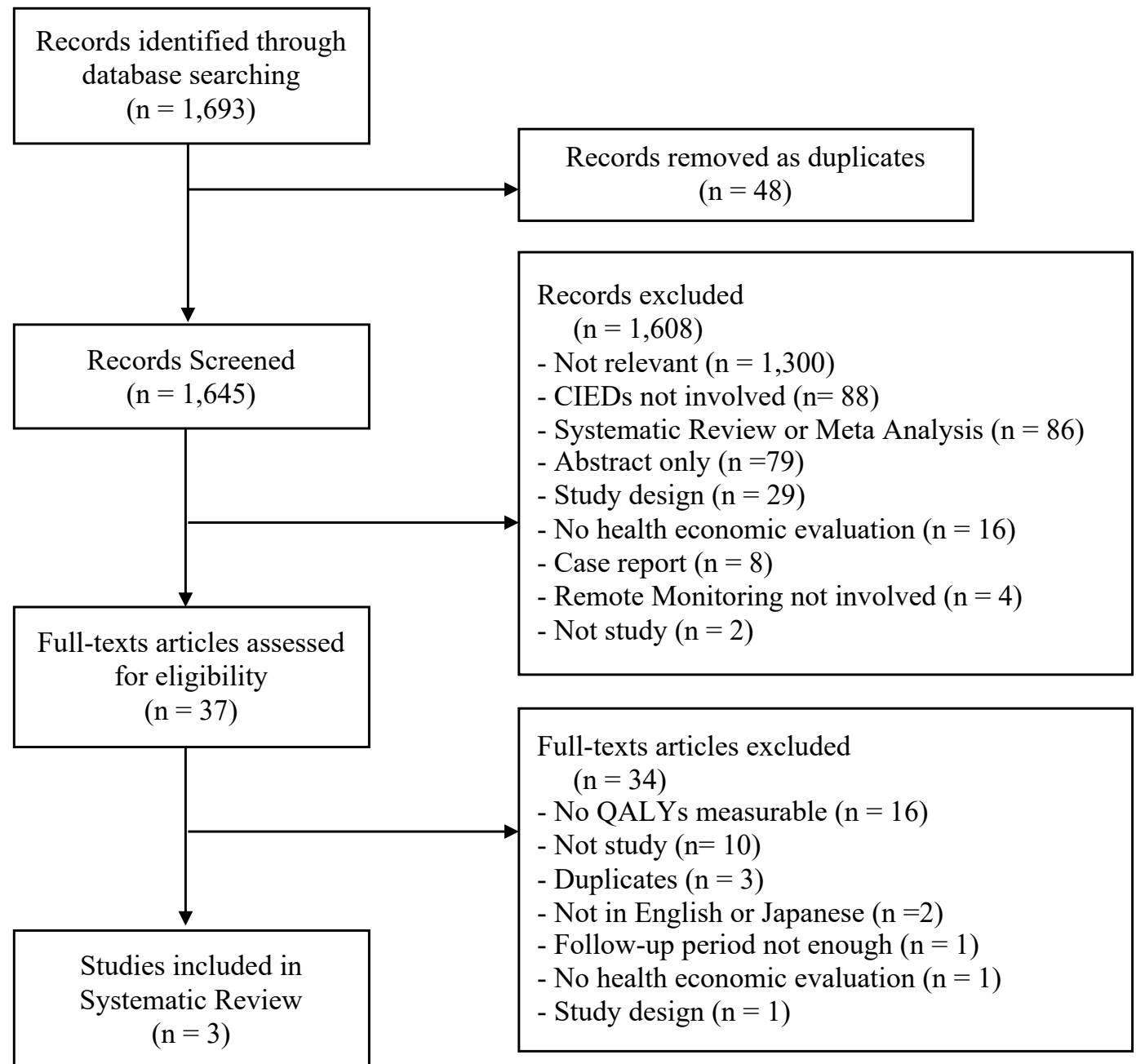

Figure 1. PRISMA flow diagram of the selection process of studies for Assessment of Cost-Effectiveness on Remote Monitoring for Cardiac Devices in Japan

\subsection{Characteristics of the Included Studies}

The main characteristics of the included studies are summarized in Table 2. Zanaboni, et al. conducted EVOLVE Study in 6 centers in Italy from 2008 to 2009 with two arms randomized. Ricci et al. also conducted TARIFF Study in Italy by having 6 centers from 2009 to 2011 with two arms non- randomized. Those two studies were designed to assess the patients implanted with defibrillators. Lopez-Villegas et al. conducted PONIENTE Trial in a single center, Poniente Hospital, in Spain from 2012 to 2013 with two arms non-randomized. This trial was designed to follow the patients implanted with pacemakers. 
Table 2. Characteristics of included studies

\begin{tabular}{|c|c|c|c|c|}
\hline \multirow[b]{2}{*}{ Study } & \multirow{2}{*}{$\begin{array}{l}\text { Author } \\
\text { Journal }\end{array}$} & Zanaboni et al., 2013 & \multirow{2}{*}{$\begin{array}{l}\text { Ricci et al., } 2017 \\
\text { Heart Rhythm }\end{array}$} & Lopez-Villegas et al., 2018 \\
\hline & & $\begin{array}{l}\text { J of Medical Internet } \\
\text { Research }\end{array}$ & & $\begin{array}{l}\text { Journal of Telemedicine } \\
\text { and Telecare }\end{array}$ \\
\hline \multirow{6}{*}{$\begin{array}{l}\text { Charac- } \\
\text { teristics }\end{array}$} & \multirow{2}{*}{ Period } & 2008 May to & 2009 Dec to & 2012 Oct to \\
\hline & & $2009 \mathrm{Jul}$ & 2011 Mar & 2013 Nov \\
\hline & Country & Italy & Italy & Spain \\
\hline & Single/Multi & 6 Centers & 6 Centers & Single Center \\
\hline & Arms & Multiple (Randomized) & $\begin{array}{l}\text { Multiple } \\
\text { (Non-Randomized) }\end{array}$ & $\begin{array}{l}\text { Multiple } \\
\text { (Non-Randomized) }\end{array}$ \\
\hline & $\begin{array}{l}\text { QALYs } \\
\text { measurement }\end{array}$ & EQ-5D & EQ-5D & EQ-5D \\
\hline \multirow{5}{*}{ Follow-Up } & Duration & 16 Months & 12 Months & 12 Months \\
\hline & In Hospital Arm & $\begin{array}{l}\text { at } 4,8,12,16 \\
\text { Months }\end{array}$ & Every 3 Months & $\begin{array}{l}\text { at } 1,6 \text {, and } 12 \\
\text { Months }\end{array}$ \\
\hline & \multirow{3}{*}{ Remote Arm } & $\begin{array}{l}\text { In Hospital: } \\
\text { at } 8 \text { and } 16 \text { Months }\end{array}$ & $\begin{array}{l}\text { In Hospital: } \\
\text { at } 12 \text { Month }\end{array}$ & $\begin{array}{l}\text { In Hospital: } \\
\text { at } 1 \text { Month }\end{array}$ \\
\hline & & & Remote: & Remote: \\
\hline & & $\begin{array}{l}4 \text { and } 12 \text { Months } \\
\text { (Alerts could be detected } \\
\text { by the continuous } \\
\text { monitoring) }\end{array}$ & $\begin{array}{l}\text { Every } 3 \text { Months } \\
\text { (Alerts could be detected by } \\
\text { the continuous monitoring) }\end{array}$ & $\begin{array}{l}\text { No Schedule } \\
\text { (Alerts could be detected by } \\
\text { the continuous monitoring) }\end{array}$ \\
\hline \multirow{12}{*}{$\begin{array}{l}\text { Patient } \\
\text { Demo- } \\
\text { graphic }\end{array}$} & $\mathbf{N}$ & 200 & 209 & 82 \\
\hline & Age & 68 & 69 & 78 \\
\hline & Male (\%) & $157(75)$ & $178(85)$ & $64(78)$ \\
\hline & NYHA I (\%) & $24(12)$ & $55(26)$ & NA \\
\hline & II (\%) & $139(70)$ & $84(40)$ & NA \\
\hline & III (\%) & 37 (19) & $66(32)$ & NA \\
\hline & IV (\%) & NA & $4(2)$ & NA \\
\hline & LVEF & 30.5 & 32.0 & NA \\
\hline & ICD (\%) & \multirow{2}{*}{$200(100)$} & $99(47)$ & - \\
\hline & CRT-D (\%) & & $110(53)$ & - \\
\hline & Pacemaker (\%) & - & - & $82(1.00)$ \\
\hline & $\begin{array}{l}\text { Primary } \\
\text { Prevention (\%) }\end{array}$ & $182(91)$ & $165(79)$ & NA \\
\hline Device & Manufacture & Medtronic & St. Jude Medical (Abbott) & Medtronic \\
\hline
\end{tabular}

\subsection{Quality Assessment of Included Papers}

Table 3 and Table 4 summarize the assessment of the quality of the studies using the Critical Appraisal Skills Programme (CASP) checklists. In general, all the studies included into this research were considered as being conducted with certain quality. 
Table 3. Quality assessment by using CASP randomized controlled trial checklist

\begin{tabular}{llllllllllll}
\hline & Q1 & Q2 & Q3 & Q4 & Q5 & Q6 & Q7 & Q8 & Q9 & Q10 & Q11 \\
\hline Zanaboni, et al. 2013 & Y & Y & Y & N & Y & Y & $*$ & $*$ & Y & Y & Y \\
\hline
\end{tabular}

Note. Q1 - Did the trial address a clearly focused issue? Q2 - Was the assignment of patients to treatments randomized? Q3Were all of the patients who entered the trial properly accounted for at its conclusion? Q4 - Were patients, health workers and study personnel 'blind' to treatment? Q5 - Were the groups similar at the start of the trial? Q6 - Aside from the experimental intervention, were the groups treated equally? Q7 - How large was the treatment effect? Q8 - How precise was the estimate of the treatment effect? Q9 - Can the results be applied to the local population, or in your context? Q10 - Were all clinically important outcomes considered? Q11 - Are the benefits worth the harms and costs? * Q7 and Q8 were not scored, and the study details are discussed on Results, Discussion, and Table 2.

Table 4. Quality assessment by using CASP cohort study checklist

\begin{tabular}{|c|c|c|c|c|c|c|c|c|c|c|c|c|c|c|}
\hline & Q1 & Q2 & Q3 & Q4 & Q5-a & Q5-b & Q6-a & Q6-b & Q7 & Q8 & Q9 & Q10 & Q11 & Q12 \\
\hline Ricci, et al. 2017 & $\mathrm{Y}$ & $\mathrm{Y}$ & Y & $\mathrm{Y}$ & $\mathrm{Y}$ & Y & $\mathrm{Y}$ & $\mathrm{Y}$ & $*$ & $*$ & $\mathrm{Y}$ & $\mathrm{Y}$ & $\mathrm{Y}$ & $*$ \\
\hline Lopez-Villegas, et al. 2018 & $\mathrm{Y}$ & Y & $\mathrm{Y}$ & $\mathrm{Y}$ & Y & $\mathrm{Y}$ & $\mathrm{Y}$ & Y & $*$ & $*$ & $\mathrm{Y}$ & $\mathrm{Y}$ & $\mathrm{Y}$ & $*$ \\
\hline
\end{tabular}

Note. Q1 - Did the study address a clearly focused issue? Q2 - Was the cohort recruited in an acceptable way? Q3 - Was the exposure accurately measured to minimise bias? Q4 - Was the outcome accurately measured to minimise bias? Q5-a - Have the authors identified all important confounding factors? Q5-b - Have they taken account of the confronting factors in the design and/or analysis? Q6-a - Was the follow up of subjects complete enough? Q6-b - Was the follow up of subjects long enough? Q7 - What are the results of the study? Q8 - How precise are the results? Q9 - Do you believe the results? Q10 - Can the research be applied to the local population? Q11 - Do the results of the study fit with other available evidence? Q12 - What are the implications of this study for practice? * Q7, Q8, and Q12 were not scored, and the study details are discussed on Results, Discussion, and Table 2 .

\subsection{Study Outcomes}

QALYs were obtained from all of the studies included into this study. Zanaboni, et al. found "that chronic HF patients wearing ICD/CRT-D followed with remote monitoring gained 0.066 QALYs more than those in the standard arm over the 16-month study period" (Zanaboni et al., 2013). Ricci et al. reported 0.02 QALYs more were obtained from Remote Monitoring Group than Standard Care Group (Ricci et al., 2017). And Lopez-Villegas, et al. put the outcome that "patients in the TM group gained 0.09 QALYs more than those in the HM group" (Lopez-Villegas et al., 2018).

\subsection{ICER Analysis}

The outcome from EVOLVO Study was calculated into ICER, combined with CIEDs Remote Monitoring by the reimbursement system in Japan. In this study, patients in the standard arm visited their hospitals 4 times during the follow-up period at 4, 8, 12, and 16 months. Therefore, the follow-up cost for the standard arm was calculated as 14,400 JPY, 3,600 JPY multiplied by 4. And patients in the remote arm visited their hospitals twice at 8 and 16 months. As it was assumed that the remote monitoring system continued to monitor the patients throughout the follow-up period, 3,200 JPY as the remote follow-up fee was multiplied by 14 by excluding two months of the hospital visits. The follow-up cost for the remote arm was calculated as 52,000JPY, 3,600 JPY multiplied by 2 and 3,200 JPY multiplied by 14. The incremental cost for Remote Monitoring, 37,600 JPY, was divided by 0.066 QALYs, which resulted in 569,697 JPY as ICER, described on Figure 2.

In the same way, the outcome from TARIFF Study was also calculated into ICER. In this study, patients in Standard Care group took in-person follow-up examinations 4 times during the follow-up period at 3, 6, 9 , and 12 months. Therefore, the follow-up cost for the standard arm was calculated as 14,400 JPY, 3,600 JPY multiplied by 4. And patients in Remote Monitoring group took in-person follow-up once at 12 months. As it was assumed that the remote monitoring system continue to monitor the patients throughout the follow-up period, 3,200 JPY as the remote follow-up fee was multiplied by 11 by excluding 1 month of in-person follow-up. The follow-up cost for the remote arm was calculated as 38,800JPY, 3,600 JPY and 3,200 JPY multiplied by 11. ICER was provided as 1,220,000 JPY based on the calculation of 24,400 JPY divided by 0.02 QALYs earned more for Remote Monitor group than Standard Care group as shown on Figure 2.

The same calculation was conducted to demonstrate ICER from the outcome of PONIENTE Trial. In this study, 
patients in the hospital monitoring group had in-office visits 3 times during the follow-up period at 1,6 , and 12 months. Therefore, the follow-up cost for the hospital monitoring group was calculated as 10,800 JPY, 3,600 JPY multiplied by 3 . And patients in the telemonitoring group had in-office visit once at 1 month. As the remote monitoring system actually continued to monitor the patients throughout the follow-up period, 3,200 JPY as the remote follow-up fee was multiplied by 11 by excluding one month of in-office visits. The follow-up cost for the telemonitoring group was calculated as 38,800 JPY, 3,600 JP and 3,200 JPY multiplied by 11. ICER was then obtained as 311,111 JPY by dividing 28,000 JPY by 0.09 QALYs gained more for the telemonitoring group than the hospital monitoring group, as indicated in Figure 2.

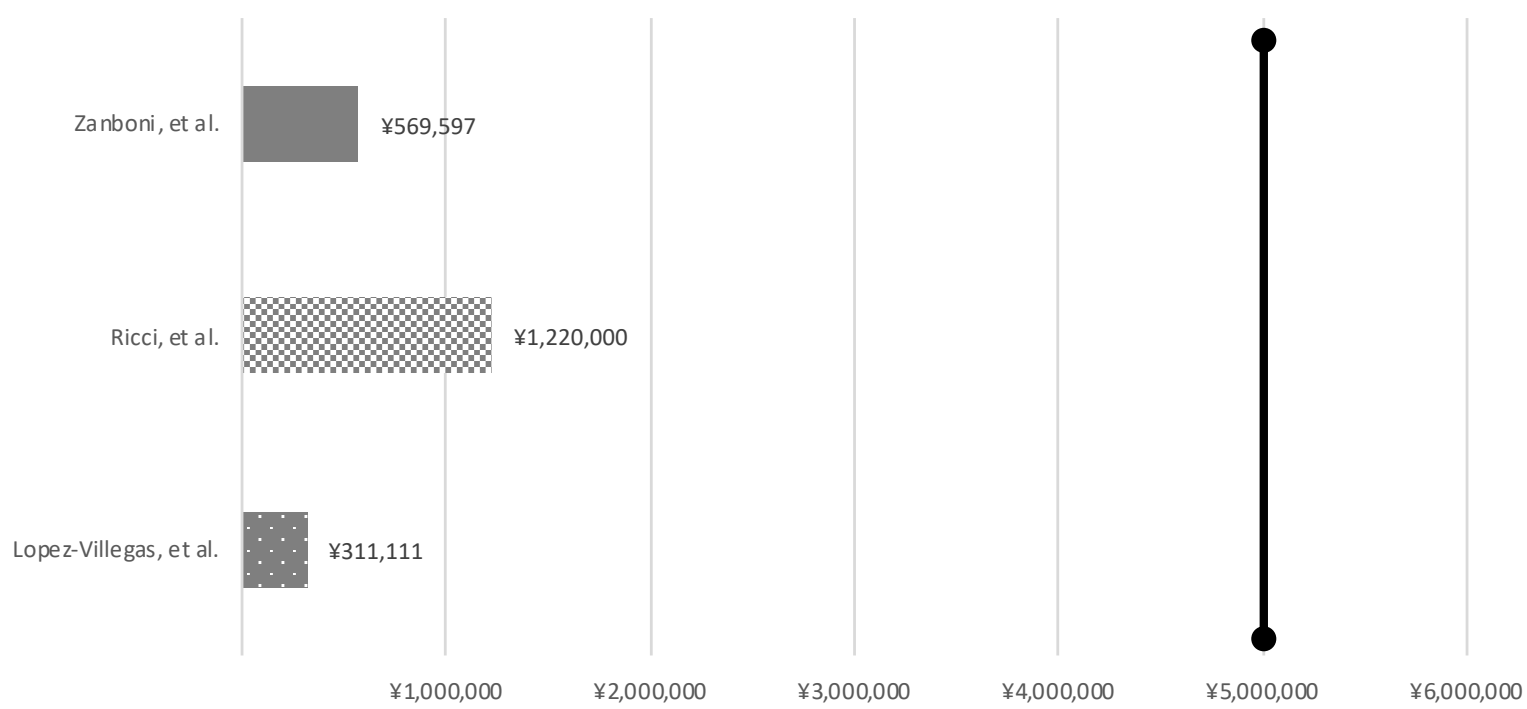

Figure 2. ICER Analysis based on QALYs from three studies included into this study

Note. Three horizontal bars indicate ICERs from each study by calculating QALYs with Follow-Up fee in Japan. The vertical black bar, 5 Million JPY, is put as ICER threshold as cost-effective set by Chuikyo.

\subsection{Sensitivity Analysis}

One-way sensitivity analysis was conducted to handle the uncertainty of the outcome by this study. Different amounts of Remote Monitoring fee per month were applied to the outcome from the three studies included into this research, as indicated in Figure 3. Remote Monitoring fee was increased to 36,000 JPY to evaluate the outcomes from different perspectives. 


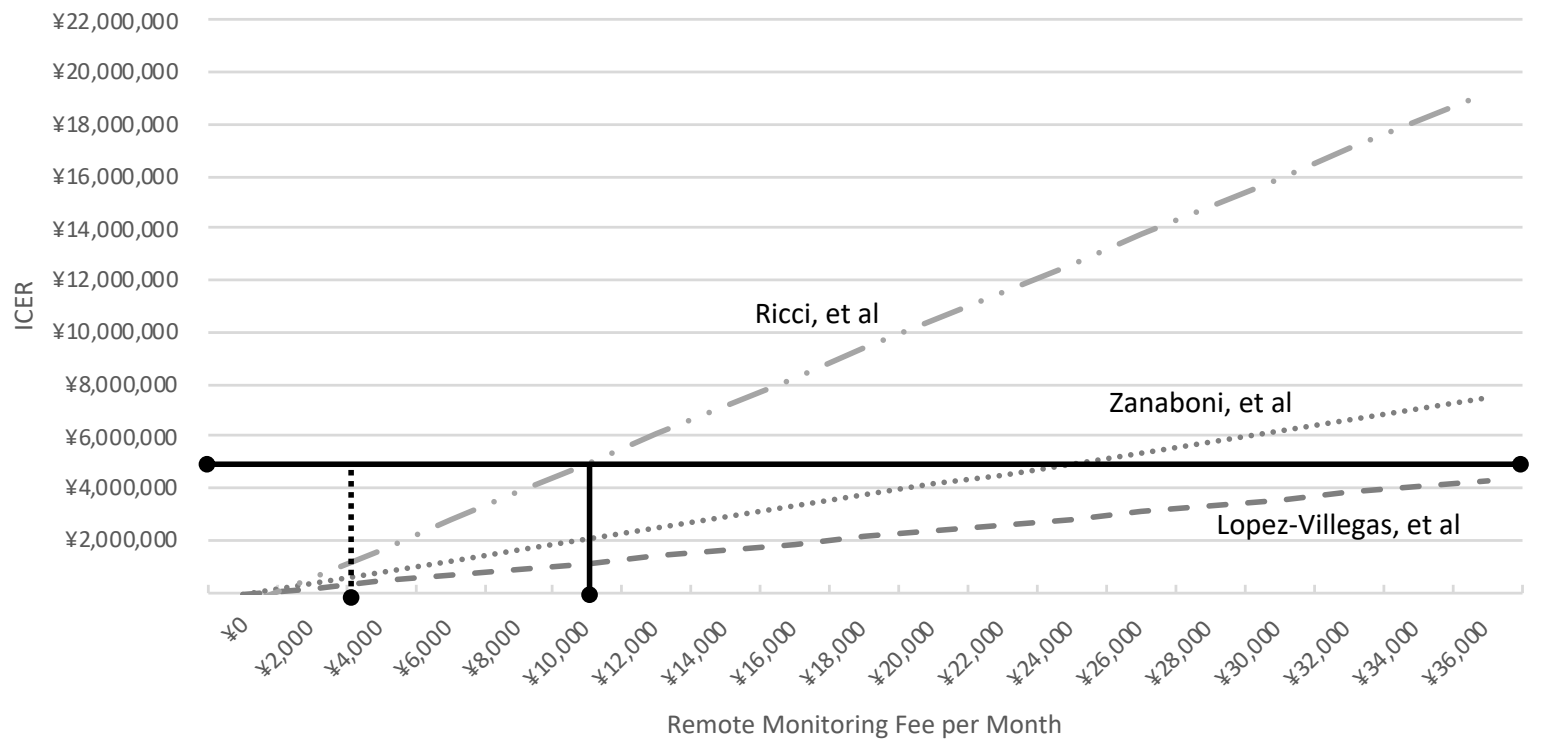

Figure 3. One-Way Sensitivity Analysis by different Remote Monitoring fee

Note. The horizontal black bar indicates ICER threshold as cost-effective set by Chuikyo. The vertical black bar as solid is the upper limit of Remote Monitoring fee per month by QALYs from all the included studies. The vertical black bar as dotted indicates Remote Monitoring fee per month, 3,200 JPY, currently reimbursed in Japan.

The guideline by Chuikyo recommends that 2.0 percent as the discount rate be applied to the cost-effectiveness analysis and the outcome by the rate be also evaluated by the sensitivity analysis. The discount rate as $2.0 \%$ annually, however, was not applied to this study, for the follow-up periods from two studies are 1 year. The guideline by Chuikyo also puts the exception that the discount rate can be omitted if the follow-up period is equal to or less than 1 year, or not long enough (Fukuda, 2015). Even one study having the longest study duration has the follow-up period with 16 months, which is not long enough to take this cost-effectiveness analysis with the discount rate.

\section{Discussion}

This research was the first attempt to analyze the cost-effectiveness of CIEDs Remote Monitoring in Japan. In this research, three studies were included into the cost-effective analysis by utilizing QALYs and ICER calculations.

There are countries that have the threshold to evaluate how much incremental cost (ICER) can be justified for the better outcome as alternative therapy or intervention. For example, the National Institute for Health and Care Excellence (NICE) in the United Kingdom puts the threshold for what is considered acceptable value ranging between GB£20,000 and GB£30,000 pounds (or approximately US\$30,000-45,000) (Dubois, 2015). The United States has a different criteria as a range from 50,000 USD to 150,000 USD (Neumann \& Cohen, 2018). For instance, American College of Cardiology and American Heart Association (ACC/AHA) categorizes services that would exceed three times GDP per capita are viewed as economically unattractive (based upon 2011 US GDP, that would be $>$ US $\$ 150,000 /$ QALY $)$ and services less than one times per capita GDP $(<$ US\$50,000) are considered cost effective (Dubois, 2015).

In Japan, the cost effectiveness has been discussed in Chuikyo under MHLW, and 5,000,000 JPY was set as the threshold by which the cost adjustment has to be considered. A pilot program about the cost effectiveness evaluation on some drugs and medical devices was conducted in 2016, and the full implementation of this cost adjustment system is further discussed in 2019 (Fukuda, 2018).

Then all the results from the included studies were compared with the threshold that Chuikyo has put for the cost-effective. As shown clearly on Figure 2, all ICERs from the included studies in this research were ranged within the threshold set by Chuikyo. At the same time, one-way sensitivity analysis was conducted by all the outcomes from the included studies as indicated on Figure 3. Two studies, EVOLVO and PONIENTE, had higher QALYs gained, which resulted in accepting the higher amount of Remote Monitoring fee, almost toward 24,000 JPY per month. On the other hand, TARIFF, with lower QALYs compared with the other studies, showed 
10,072.73JPY as the upper limit of Remote Monitoring fee per month against the threshold set by Chuikyo. While these results are considered, anyway, it can be said that the current fee as 3,200 JPY per month can be accepted within the range of ICER threshold as cost-effective.

ICER analysis does not have the long story yet in Japan, and as described in this chapter, the cost effectiveness evaluation just started as a pilot program in 2016. Japan is sometimes characterized as the super-aging society with the high life expectancy. Because the health insurance system is public and all the medical costs are set by the government, MHLW, the longer life expectancy is bringing the higher medical spending overall. This trend put much more attention on the importance of the cost-effectiveness evaluation of the medical costs.

This study has been done along with the guidelines by Chuikyo, and can be considered to put an additional case example that ICER can be one of rationale approaches to evaluate the reimbursement items. ICER is the evaluation system accepted in many countries, and more cases like this study should be performed to show how the cost-effectiveness evaluation can be valid in Japan as well.

There are several limitations in this study. This research includes three studies from patients with defibrillators or pacemakers. As indicated on Table 2, the patient characteristics from defibrillators and pacemakers are different as the implant indications for each of devices are not the same. As the reimbursement amount for those devices have no difference in terms of cost per visit, the cost analysis from this study put no distinctions between them, but the cost analysis based on each device separately might bring more insight.

Remote Monitoring systems from different manufactures have their own algorithm and features to detect alert and send notifications based on their own frequencies although the basic specifications do not vary so much. The studies included into this study have the systems from two manufactures indicated on Table 2. Also, the enrollment and follow-up took place almost 10 years ago, and we are seeing a lot of improvements around CIEDs themselves and Remote Monitoring system. The assessment by the latest version of devices and systems from various manufactures with the setting standardized might provide more robust outcomes.

This study was designed to focus on the reimbursement fee as medical cost for CIEDs patient managements so that the analysis can be simplified. As such patient managements may involve the other medical costs, more comprehensive analysis might be helpful to obtain better understanding of the cost effectiveness around overall CIEDs patient management.

This study has been implemented as the graduate school research. Due to the limited time and resources, the systematic review has been done by only one reviewer. In order to mitigate the bias during the review process, the reviewers should consist of two or more in general.

\section{Conclusion}

This study was designed and carried out to analyze the cost-effectiveness about CIEDs Remote Monitoring that have been recommended under the guidelines or the statement by the societies across the world. Three studies included after the systematic search and review based on certain criteria that was pre-defined showed considerably low ICERs from the calculation with QALYs and the reimbursement amount from Japan health insurance system. These ICERs were further demonstrated as ranged within the threshold set as cost-effective by Chuikyo. Considered this study as a valid approach as the cost-effective evaluation in Japan as well, this kind of analysis should be performed more areas, both drugs and medical devices, so that the medical cost can be assessed in more systematic way.

\section{Acknowledgements}

This research was conducted under the supervision of Professor Takao Tashiro at Open University of Japan, and it could not be completed without his instructions. The study design was first presented during the 13th Annual Conference of Japan Health Economics Association. The chair was Dr. Rei Goto from Keio Business School and the commentator was Dr. Noriko Sasaki from Kyoto University. Dr. Sasaki showed the basic ideas about how to take the cost-effectiveness evaluation about medical devices, which became the foundation of this research. Professor Shigeru Tanaka from Keio University hosted a seminar camp, which gave another opportunity for me to present the study design in front of the participants. Finally, my family members put great supports on my efforts for this review to be completed.

\section{Competing Interests Statement}

This author declares that there are no competing or potential conflicts of interest. 


\section{References}

Critical Appraisal Skills Programme (CASP). (2018a). CASP Cohort Study Checklist. Retrieved from https://casp-uk.net/wp-content/uploads/2018/01/CASP-Cohort-Study-Checklist_2018.pdf

Critical Appraisal Skills Programme (CASP). (2018b). CASP Randomised Controlled Trial Checklist. Retrieved from https://casp-uk.net/wp-content/uploads/2018/01/CASP-Randomised-Controlled-Trial-Checklist-2018. pdf

Dubois, R. W. (2015). Cost-effectiveness thresholds in the USA: are they coming? Are they already here? Journal of Comparative Effectiveness Research, 5(1), 9-12. https://doi.org/10.2217/CER.15.50

Fukuda, T. (2015). Analysis Guidelines of Cost-Effectiveness Evaluation under Central Social Insurance Medical Council. Tokyo, Japan.

Fukuda, T. (2018). A Pilot Program of Implementing Health Technology Assessment to Decision Making in Japan. Jpn J Pharmacoepidemiol, 23(1), 3-10. https://doi.org/10.3820/jjpe.23.3

Japanese Heart Rhythm Society. (2016). Remote Monitoring Statement on Cardiac Implantable Electronic Devices. Retrieved May 9, 2018, from http://new.jhrs.or.jp/guideline/statement201607_01/

Kurita, T., \& Nogami, A. (2019). 2018 JCS/JHRS Guideline on Non-Pharmacotherapy of Cardiac Arrhythmias.

Kusano, K. (2017). Evolution of Arrhythmia Treatment. Latest Information of Cardiovascular Treatment 2016.

Lopez-Villegas, A., Catalan-Matamoros, D., Robles-Musso, E., Bautista-Mesa, R., \& Peiro, S. (2018). Cost-utility analysis on telemonitoring of users with pacemakers: The PONIENTE study. Journal of Telemedicine and Telecare, 1-9. https://doi.org/10.1177/1357633X18767184

Neumann, P. J., \& Cohen, J. T. (2018). QALYs in 2018-Advantages and Concerns. JAMA. https://doi.org/10.1001/jama.2018.6072

Ponikowski, P., Voors, A. A., Anker, S. D., Bueno, H., Cleland, J. G. F., Coats, A. J. S., .. Van Der Meer, P. (2016). 2016 ESC Guidelines for the diagnosis and treatment of acute and chronic heart failure. European Heart Journal, 37(27), 2129-2200m. https://doi.org/10.1093/eurheartj/ehw128

Ricci, R. Pietro, Vicentini, A., D’Onofrio, A., Sagone, A., Rovaris, G., Padeletti, L., ... Santini, M. (2017). Economic analysis of remote monitoring of cardiac implantable electronic devices: Results of the Health Economics Evaluation Registry for Remote Follow-up (TARIFF) study. Heart Rhythm, 14(1), 50-57. https://doi.org/10.1016/j.hrthm.2016.09.008

Shoda, M. (2012). Life Threatening Arrhythmia and Remote Monitoring. Japanese Journal of Electrocardiology, 32(4), 410-418. https://doi.org/10.5105/jse.32.410

Slotwiner, D., Varma, N., Akar, J. G., Annas, G., Beardsall, M., Fogel, R. I., .. Yu, C. M. (2015). HRS expert consensus statement on remote interrogation and monitoring for cardiovascular implantable electronic devices. Heart Rhythm, 12(7), e69-e95. https://doi.org/10.1016/j.hrthm.2015.05.008

Zanaboni, P., Landolina, M., Marzegalli, M., Lunati, M., Perego, G. B., Guenzati, G., ... Masella, C. (2013). Cost-utility analysis of the EVOLVO study on remote monitoring for heart failure patients with implantable defibrillators: Randomized controlled trial. Journal of Medical Internet Research, 15(5), e106. https://doi.org/10.2196/jmir.2587

\section{Copyrights}

Copyright for this article is retained by the author(s), with first publication rights granted to the journal.

This is an open-access article distributed under the terms and conditions of the Creative Commons Attribution license (http://creativecommons.org/licenses/by/4.0/). 